\title{
Neural stimulation systems for the control of refractory epilepsy: a review
}

\author{
Matthew D. Bigelow and Abbas Z. Kouzani ${ }^{*}$ (D)
}

\begin{abstract}
Epilepsy affects nearly 1\% of the world's population. A third of epilepsy patients suffer from a kind of epilepsy that cannot be controlled by current medications. For those where surgery is not an option, neurostimulation may be the only alternative to bring relief, improve quality of life, and avoid secondary injury to these patients. Until recently, open loop neurostimulation was the only alternative for these patients. However, for those whose epilepsy is applicable, the medical approval of the responsive neural stimulation and the closed loop vagal nerve stimulation systems have been a step forward in the battle against uncontrolled epilepsy. Nonetheless, improvements can be made to the existing systems and alternative systems can be developed to further improve the quality of life of sufferers of the debilitating condition. In this paper, we first present a brief overview of epilepsy as a disease. Next, we look at the current state of biomarker research in respect to sensing and predicting epileptic seizures. Then, we present the current state of open loop neural stimulation systems. We follow this by investigating the currently approved, and some of the recent experimental, closed loop systems documented in the literature. Finally, we provide discussions on the current state of neural stimulation systems for controlling epilepsy, and directions for future studies.
\end{abstract}

Keywords: Refractory epilepsy, Closed loop stimulation, Biomarkers

\section{Introduction}

Epilepsy is a serious and debilitating disease, affecting more than 50 million people worldwide [1]. Of these, approximately one third suffer from refractory epilepsy, or epilepsy that cannot be controlled by medication. The uncontrolled nature of this disease means those affected sustain different levels of secondary injuries, illnesses, social dysfunction, and decreased life expectancy. Patients often experience a reduced quality of life (QOL) and the social stigma surrounding epilepsy often leads to discrimination and social rejection [2].

Resective surgery and newly developed or experimental drug treatments have been found to help around 65\% of these patients [3]. For nearly 6.5 million sufferer's, however, alternative treatments such as neural stimulation can be an effective, though potentially underutilised form of control. Neural stimulation has traditionally been in open loop form however, the use of closed loop systems, such as Cybernetics's responsive neuro stimulation (RNS) or Neuropace's vagal nerve stimulation

\footnotetext{
* Correspondence: kouzani@deakin.edu.au

School of Engineering, Deakin University, Geelong, Victoria 3216, Australia
}

(VNS), offers the potential for enhanced timing of delivery over open loop systems. However, despite this potential, when comparing to open loop systems, there has not been significant improvement in responder rates from those with a greater than $50 \%$ reduction in seizures using these methods. Leveraging research to provide an improved online prediction or detection method and integrating it into a system that can deliver effective, timely stimulation could be a step forward in in the batthe to provide relief to these patients.

A successful closed loop system requires the interfacing of two subsystems: (i) a biosensing system that couples with signal classification algorithms to determine the epileptic state of a patient, and (ii) a neural device that provides effective control of seizures. Furthermore, these subsystems need to be interfaced using an appropriate communications protocol and a control algorithm to deliver an effective neural treatment using closed loop techniques. Several studies have shown that there are alternative options available in terms of biomarkers and classifiers that can be used to determine ictal and pre-ictal periods than those currently used in

(C) The Author(s). 2019 Open Access This article is distributed under the terms of the Creative Commons Attribution 4.0 International License (http://creativecommons.org/licenses/by/4.0/), which permits unrestricted use, distribution, and 
approved systems. Furthermore, there are alternative non-invasive neural stimulation systems other than the invasive methods currently approved for medical use. Therefore, in this paper, we have conducted a systematic literature search on Google Scholar, Pubmed and Medline, and IEEE Xplore using various combinations of keywords including epilepsy, biomarkers, prediction, detection, closed loop, open loop, neural and stimulation. Using the information derived from these searches we give a brief description of epilepsy, some of epidemiology surrounding it, and a look into what considered as refractory epilepsy. We investigate, and give examples of, some of the more important, biomarkers that can be used in the prediction and detection of seizures. These descriptions include the method of feature extraction and the algorithms used to classify pre-ictal and ictal periods. The paper then explores neurostimulation methods both currently in use and under investigation. These methods are categorised into invasive and noninvasive approaches showing that non-invasive approaches have the potential for further development in the future. Next, current approved closed loop systems are highlighted and described, followed by a description of some alternative methods that have been investigated to improve the closed loop systems. Finally, discussions are presented on the current state of the available systems, and suggestions are made for possible future directions that studies could take to improve the current systems. These suggestions could improve the overall quality of life of those suffering from this debilitating and potentially life-threatening condition.

\section{Epilepsy}

\section{Description and epidemiology}

Epilepsy is described [4] as a "disease of the brain characterized by an enduring predisposition to generate epileptic seizures, and by the neurobiologic, cognitive, psychologic, and social consequences of this condition". Under normal circumstances, clusters of nerve cells called neurons interact electrically with other neurons to produce normal motor and non-motor actions by electrical and chemical signals. Seizures are caused by clusters of these neurons, signalling excessively and hyper synchronously [5]. Epileptic seizures are a consequence of these neurons producing these signals rapidly, and simultaneously manifesting in involuntary and spontaneous movements, emotions and behaviours, and sufferers can either be in a conscious or unconscious state [6-8]. This abnormal neuronal firing is what distinguishes epileptic seizures from nonepileptic events [5].

In 2015, epilepsy was responsible for more than 8 million years of life lost, and 14.6 million disability-adjusted life-years lost [9], both these figures are more than 4 times that of Parkinson's disease. Despite technological advancement in traditional methods of treating epilepsy, including medication based treatments and surgical techniques, standardised mortality ratio and mortality rates have only decreased slightly in people diagnosed with epilepsy over the last 50 years [10].

\section{Refractory epilepsy}

Refractory or drug resistant epilepsy accounts for about one third of all epilepsy cases [2]. ILAP's definition of drug resistant epilepsy is: "drug resistant epilepsy may be defined as failure of adequate trials of two tolerated and appropriately chosen and used anti-epileptic drug (AED) schedules (whether as monotherapies or in combination) to achieve sustained seizure freedom" [11]. The uncontrolled nature of the disease can present those affected with increased experiences of additional illnesses. It is common for patients to experience psychological dysfunction which can result in reduced quality of life (QOL) and decreased life expectancy. Social stigmatisation commonly surrounds epilepsy and can lead to discrimination and social rejection [2].

The most common form of refractory epilepsy is temporal lobe epilepsy (TLE). TLE is a focal onset epilepsy that can become more generalized as the seizure progresses. There are two types of TLE, medial and neocortical. Medial, the most common, involves the internal structures of the temporal lobe, often beginning in the hippocampus or structures surrounding it. Neocortical is associated with the outer structures of the temporal lobe [12]. Treatment of this type of epilepsy ranges from continued medical treatment that includes the use of new or experimental drugs, which is estimated to eventually help approximately $15 \%$ of patients, and surgery including resective and non-resective procedures which leave an estimated $57 \%$ of patients seizure free [3]. The remaining $28 \%$ of drug-resistant epilepsy sufferers are without effective treatment and potentially benefit from alternative treatments such as neurostimulation.

\section{Epilepsy seizure prediction and detection}

Identifying and capturing information of biometric markers of epilepsy is an area that could have a profound effect on expediating our knowledge and potentially engineering solutions for those with drug-resistant epilepsy. Clinically, little is still known about epilepsy biomarkers, for several reasons [13], however, research into identifying potential biomarkers for use in prognosis, diagnostic and seizure detection or prediction systems has been a focus of research for several decades $[13,14]$.

Most seizure tracking is currently conducted by patient recall and family experience, which is found to be unreliable due to the patient's awareness during seizures [15]. The ability to autonomously predict or track 
seizures potentially have a large effect on an epileptic patient and their family's quality of life. It has the potential to fill the gap for those drug-resistant epilepsy sufferers that have found no respite either in new AEDs or from surgical attempts to relieve their seizures. Neural stimulation procedures could benefit greatly from the ability to predict or track seizures potentially totally relieving those patients of symptoms.

Introducing warning systems, utilising the ability of biomarkers to identify seizures and wireless technology, could potentially lower mortality rates. Alerting patients, relatives or caregivers, that a seizure is imminent would allow them to take appropriate steps to limit the risk of further injury during high seizure risk periods. Moreover, continuous recording of biomarker activity can potentially help researchers with important clinical information that is not available to them by traditional reporting and tracking methods.

However, the balance between sensitivity and accuracy in seizure identification or prediction is an important consideration for any model. The trade-off between the two must be considered in the engineering of a closed loop neural stimulation system. Other considerations for biomarkers must also include the invasive/non-invasive requirements of the device. Invasive devices have risks including infection, rejection or procedural dangers, while consideration of non-invasive devices include the wearability, comfort and robustness of the device and the social stigma associated with wearing certain devices.

\section{Electroencephalogram (EEG) and electrocorticography (ECoG)}

EEG or ECoG is perhaps the most researched form of seizure prediction and detection, and is part of the gold standard method used in clinical seizure detection. Electrical activity of the brain measured by EEG or ECoG, also known as intercranial EEG (iEEG), has produced potentially useful results in seizure prediction and detection. The combination of EEG/Video monitoring is the gold standard in seizure detection, used by clinicians internationally for epilepsy diagnosis [16]. EEGs is conducted by non-invasive methods consisting of placing several electrodes directly onto the scalp, usually 19 recording, one ground and a reference electrode in accordance with the international $10-20$ system. On the other hand the ECoG is an invasive method that requires electrodes to be placed directly onto the brain's surface [17]. An Australian ground-breaking, first-in-man, proof of concept study revealed sensitivities between 65 and $100 \%$ using intercranial EEG methods for seizure prediction in 15 patients, however, the algorithm used was unspecified in the literature [18] .

Both methods result in a graphical record that is a measure of electrical activity in terms of voltage fluctuations in different areas of the brain. To allow seizure detection or prediction, these measurements are processed by taking several steps to extract features, analyse those features and then classify the signal appropriately. There are, however, significant differences in the steps required to complete seizure detection than those required for prediction [19] as shown in Fig. 1. A significant challenge with these methods is that seizure patterns can differ significantly from patient to patient, therefore any algorithm must be able to adapt to an individual's pattern to successfully classify events $[17,20]$.

While methods used to detect seizures or predict ictal and preictal states have been classified as linear or nonlinear methods, [17] suggests that due to a significant quantity of recent research focused on non-linear methods, an improved means to classify them is by their transform domain of operation.

\section{Seizure detection}

In 2013, an 8-channel, ultra-low power, system on chip (SoC) seizure detection system was proposed by [20]. The system takes advantage of up to $120 \mathrm{~s}$ of stored EEG data, extracts features and then uses a machine learning seizure classification processor. The approach estimates the signal sub-bands, as opposed to the signal as a whole, to build a feature vector to detect seizure and non-seizure periods. The processor was "tested with the CHB-MIT database, and the SoC was verified with a rapid eye blink test, which shows typical accuracy of $84.4 \%$ with $2.03 \mu \mathrm{J} /$ classification energy efficiency" [20].

Tessy [21] used two time-domain features extracted from intracranial and scalp EEG databases. The method involved using both line length and energy feature extraction followed by a $\mathrm{K}$ nearest neighbour learning algorithm as the classification method for seizure detection. The method was described as simple, with lower costs and faster results when compared to more computationally expensive methods. It was found to give similar high degrees, nearing $100 \%$, accuracy, specificity and sensitivity. It must be noted however that the datasets used for these methods were artefact free and would need to be tested under real world conditions [21].

Other methods include extracting statistics such as zero-crossing, entropy, mean or variance from time domain signals, and employing these in detection algorithms for the detection of seizures [17]. Alternatively, it has been suggested that wavelet features are the most commonly used features for seizure detection, however, more processing steps are required to extract features from wavelet coefficients, making it more computationally expensive than other methods [22]. An example of leveraging the wavelet domain was proposed in [23] when the authors produced a seizure detection system that uses only seven features obtained from, what is 


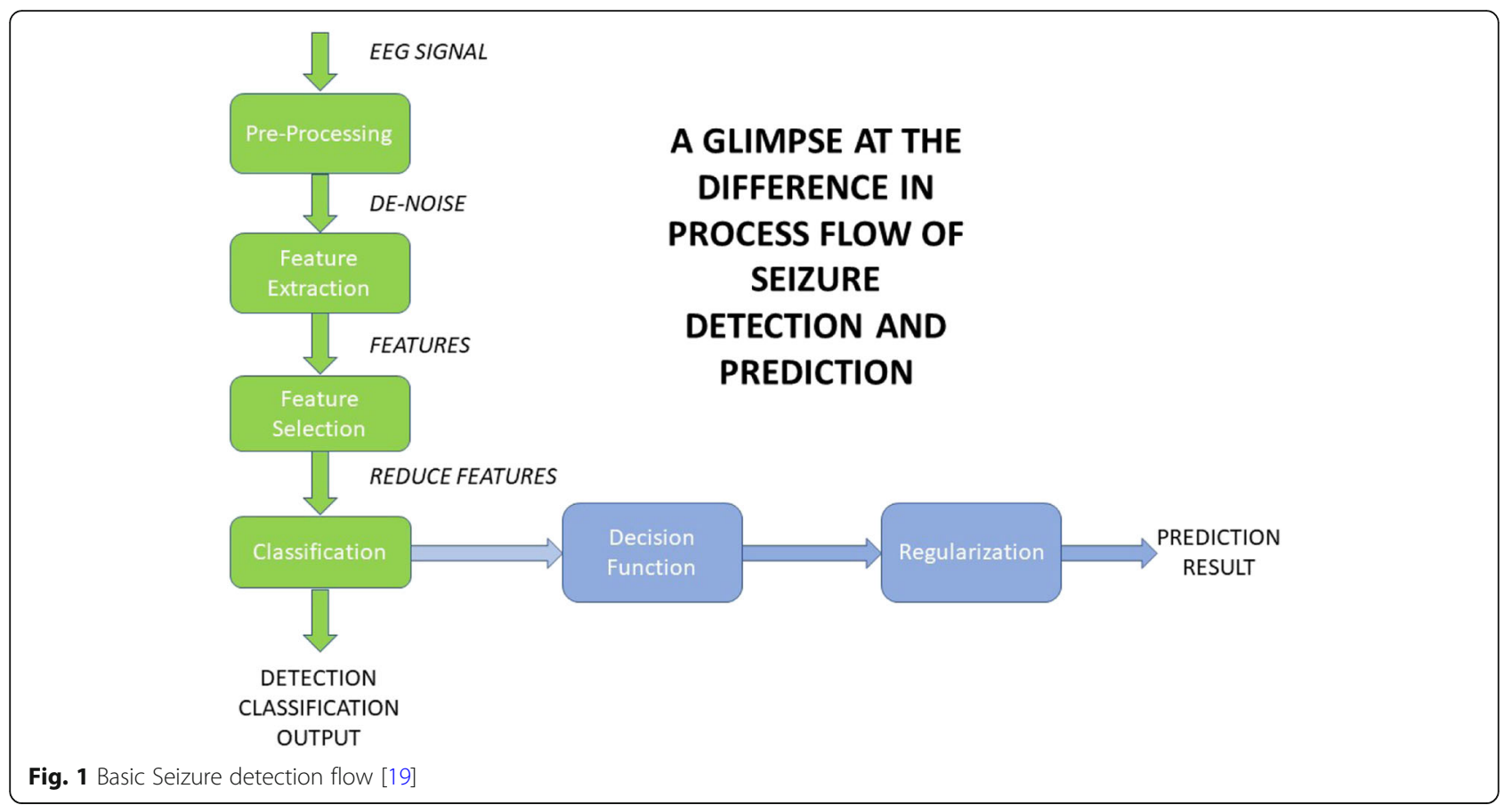

described as, a new class of minimally mean squared frequency localized, orthogonal wavelet filter bank designed for minimising the frequency spread. The method is shown to produce accuracies near $99 \%$ with sensitivities and specificity greater than $98 \%$.

\section{Seizure prediction}

Seizure prediction can be observed as a detection of the pre-ictal state that requires a considerable inter-ictal dataset to produce usable results [24]. proposed the use of a moving window analysis of positive zero-crossing intervals from scalp EEGs as the basis of the features. Specifically, using sets of reference distributions, the algorithm monitors changes over time of the distribution of positive zerocrossings. Using EEG data taken from 3 patients, it was found that the algorithm predicted 12 out of 14 seizures (86\%) with an average time to seizure of $20.8 \mathrm{~min}$ and with a false positive rate (FPR) of 0.12 per hour [24].

In another approach, following pre-processing to remove certain artefacts, power features were extracted from 9 different spectral bands from 6 electrodes in each 20-s long half overlapping window. The method used a patient-specific classification algorithm based on a costsensitive support vector machine that could distinguish preictal from interictal states with a high sensitivity of near $80 \%$. Also, importantly, the sensitivity was achieved with a zero FPR [25].

Behnam [26] proposed a real time prediction algorithm using recursive least squares (RLS) filtering. They proposed a real-time seizure prediction algorithm that included a Bayesian classifier and Hunting search to choose optimal features to train an offline seizure detection algorithm. A multi-layer perceptron classifier was trained with the pre-mentioned optimal features for an online detection algorithm, the RLS Filter was then applied to consecutive samples to gain online prediction. The algorithm was found to have an accuracy of $86.56 \%$, precision rate of $86.53 \%$, recall rate of $97.27 \%$, FPR of $2.15 \times 10^{-3}$ per hour with a prediction time of $6.64 \mathrm{~s}$.

Moreover, frequency methods were used in [27] in a field-programmable gate array (FGPA) based system for real time seizure prediction. A feature vector was extracted from six channels of iEEG data using a threesecond sliding window with 2 seconds overlap, and computing the average cross spectral density of each pair of windowed signals, and the autospectrum density of each signal using Welch's method. The brain was then modelled as a fully connected graph represented as a matrix using the resulting signals to weight the edges with the magnitude squared of a coherence estimate. The matrix was normalised and transformed to a 'number of electrodes' $\times 1$ feature vector, representing the window by computing an element of its eigenvector centrality. The feature was then classified using a one-class support vector machine that classifies each feature as either normal or an outlier. This resulting output was used to further calculate the probability that a seizure is occurring by comparing a maximum likelihood estimate to a patient specific probability threshold. The algorithm successfully predicted 11 of 11 seizures with an average FPR of 3.9 per hour, at an average of $3.6 \mathrm{~min}$ before seizure onset [27]. 
In [28] investigated several seizure prediction and detection algorithms using wavelet components. The most successful method, when tested on adult seizure data, firstly used multiscale principal component analysis to denoise the EEG data, then wavelet packet decomposition was used to decompose the signal into wavelets. Four features were extracted from the resulting subbands including: (i) the mean of the coefficients' absolute values in every sub-band, (ii) the average power of the coefficients in every sub-band, (iii) the standard deviation of the coefficients in every sub-band, and (iv) the ratio of absolute mean values of adjacent sub-bands. These features, using a random forest classifier, obtained an accuracy and sensitivity of over $99 \%$ for both seizure databases used in experiments. Although these results are high, the wavelet domain analysis can require high computational cost.

\section{Electrocardiogram methods}

Epilepsy is believed to affect the cardiovascular function, and has been a focus of research over the last decade into the possibility of using heart rate variability (HRV) as a means of seizure prediction. The disproportionate neural activity that occurs in the preictal period has also been found to affect the area of the nervous system that affects the control of autonomous bodily functions including HRV [29].

Heart rate variability deals with the analysis of intervals between the beats of the heart. These intervals, known of RR intervals can be tracked and collected using an ECG to produce RR time series data [30]. This statistical information can then be leveraged to become the basis of analysis to predict oncoming seizures using methods like those discussed in EEG seizure prediction. This beat to beat analysis generally consists of several stages including: (i) pre-processing/filtration, (ii) feature extraction, (iii) feature selection stage, (iv) classification stage, and (v) validation stage. These stages consist of similar filtering and machine learning techniques as those discussed previously. Similarly, they are generally patient specific, requiring datasets to be established [31].

However, an advantage of ECG as a use for extracting a prediction or detection biomarker is that the noninvasive version is concealable for everyday use, thus it can potentially reduce the social stigma that would be associated with the wearing of an EEG monitor. In fact, [32] proposed the design of a wearable telemeter, compatible for RR Interval measurement that can operate for up to $10 \mathrm{~h}$ with the ability to store data in a smartphone via Bluetooth wireless transmission.

\section{Seizure prediction}

Fujiwara [33] proposed a prediction method that uses multivariate statistical process control (MSPC), using features extracted from the HRV data. The method uses 8 features from the time and frequency domains and then uses MSPC to identify the preictal period as an anomaly from the usual interictal period. Tests were conducted on the clinical data from 14 patients that included $57 \mathrm{~h}$ of interictal data in which 8 patients had 11 awakening preictal episodes. Although it was noted that HRV individuality was important for prediction success, the proposed method demonstrated that $91 \%$ of preictal episodes could be predicted before seizure onset, with an FPR of approximately 0.7 times per hour where the preictal period was defined as a period within $15 \mathrm{~min}$ before a seizure occurs. It was suggested that although this is a higher FPR than that demonstrated by EEG methods, the method had greater advantage from an everyday use standpoint [33].

In [29] they proposed a prediction algorithm using the HRV analysis, and anomaly detection one-class support vector machines (SRV). The method uses extraction of the same 8 features from both the time and frequency domains of a window size, then analyses a matrix of eigenvalues and eigenvectors to produce 9 eigenvalues and principal components to be used in the classifier. The classifier separates the dataset into interictal and preictal parts, the interictal parts consist of the last 8 moving windows before 2 preictal intervals. Since there is a large amount of data for the interictal period, an anomaly detection approach is used and considers any interval that is not interictal, as preictal. The one-class SRV is used as the anomaly detection machine, it is an unsupervised machine learning algorithm that classifies new data as similar or different from the training set [29]. Tests of differing sized window periods were conducted on 31 sleeping patients with both generalised and focal onset seizures that had a total of 232 preictal intervals with a total of $85 \mathrm{~h}$ of interictal periods. Results showed that the optimal window size for prediction was $400 \mathrm{~s}$ with $100 \%$ sensitivity, a specificity rate of $92 \%$, and precision rate of $92 \%$. An F1 score of $92 \%$ was achieved with a fraction error length of 5\% [29].

Another study analysed $8 \mathrm{HRV}$ features to predict seizures from 7 patient's data. The analysis results showed that linear and non-linear HRV features, such as mean heart rate, the relationship between SD1 (describes beatto-beat rapid changes) and SD2 (describes long-term beat-to-beat changes), and the relationship between high frequency and low frequency components all changed during seizure episodes indicating that these parameters are useful in seizure detection. Moreover, a seizure prediction algorithm was developed where non-linear and linear features were extracted from uniform segment lengths and compared using a patient-specific threshold to previous segments. The algorithm resulted in $88.3 \%$ sensitivity and $86.2 \%$ specificity [34]. 
In a novel approach, [31] proposed a method for a non-patient specific solution tested on 18 recordings from 14 patients. The approach involved using RR time series data that had undergone specific pre-processing techniques and was windowed with $50 \%$ overlapping. For every window, 112 HRV features were extracted and an SVM with radial basis function kernel was used as a classifier. The data was classified into two classes, interictal or preictal. The results showed that a specificity value of 0.7252 and sensitivity of 0.7252 could be obtained for a window size of $7.8 \mathrm{~min}$ for which the features are calculated and $25.8 \mathrm{~min}$ for the preictal period.

\section{Seizure detection}

As described previously, [34] describes features that can be used to distinguish seizure from non- seizure periods. Their method also specifies that the detection can be seen 5-10 min before the seizure onset, doubling as a short delay prediction method.

Similarly, [35] proposed a method of detection using a modified cardiac sympathetic index (CSI), based on the Lorenz plot. To consider everyday life activities, baseline exercise testing was included in the study to ensure that any positive results of testing could be compared to normal HRV episodes expected to occur in everyday life. The group tested four different methods of HRVanalysis with differing window lengths for each method to establish the best method. A patient specific threshold of $105 \%$ above maximum heart rate (including exercise) at any interictal period was used to establish seizure detection. The modified CSI with a window length of $100 \mathrm{~s}$ detected all seizures for 13 of the 17 patients, with either generalised or focal epilepsy, within a mean time of $16 \mathrm{~s}$ after onset ranging between $6 \mathrm{~s}$ before, and until $50 \mathrm{~s}$ after seizure onset time [35].

Fritz [36] showed that ictal-onset tachycardia occurred in more than $86 \%$ of 145 seizures of different epilepsy origin. Many cases also showed that heart rate increases occurred preceding EEG seizure onset. However, heart-rateincrease occurrence and amount differ significantly for different epilepsy types, length of effect and also general specificity of patients such as age and gender [37]. Further studies have shown that the FPR can drop significantly and more than $88 \%$ of seizures can be detected using an SVM classifier based on heart rate data alone [38].

\section{Accelerometery}

An accelerometer (ACM) is a sensor that can track movement in the $\mathrm{x}, \mathrm{y}$ and $\mathrm{z}$ plane. Accelerometry is useful in the detection of seizures, however, the seizures must take some physical form such as myoclonic, tonicclonic or some other form of motor seizure. Other sensors such as gyros and magnetos, useful in measuring different forms of movement, can be used for detecting other seizure types and have their place in detecting movement associated with seizures [16].

Most detection systems use multiple accelerometers to ensure all areas that are potentially affected by seizures can be recorded. Recognising ACM patterns during seizures is relatively easy to distinguish by eye due to stereo typical patterns in different forms of motor seizure. However, the difficulty is finding the correct parameters to use in an automated algorithm [39]. A study conducted in 2005 [39] showed that $95 \%$ of seizures had stereotypical waveforms in the ACM-signals that could be distinguished by human observers.

Moreover, [39] also proposed a method utilising a model based matched wavelet (MOD) for detecting myoclonic seizures in ACM data with around $80 \%$ sensitivity and a positive prediction value (PPV) near 0.15 . Furthermore, a method was proposed using a combination of 5 features extracted from accelerometry data that had an $83 \%$ sensitivity and a PPV of 0.35 for detecting tonic seizures in patients with severe epilepsy. However, it must be noted that $42 \%$ of the false alarms where seizures of another type [40].

An Australian study in using time domain features in controlled conditions reported an algorithm with 100\% sensitivity with very few false positives (FP), however, the algorithm only considered seizure with $>20$ s duration and was not validated in home conditions [41].

Other methods use as a wireless sensor network, and classification using the K-nearest neighbour (KNN) technique that showed promising results under normal daily conditions [42]. Other proposals include distinguishing nocturnal in-bed movements from epileptic seizures based on a hidden Markov model (HMM) [43]. Tests run on simulated epileptic seizures suggest that generalized models may be obtained using principal component analysis (PCA) and local PCA as feature extraction techniques using KNN classifiers [44], and an algorithm based on a Bayesian approach using HMM for statistical modelling tested on patients during the night, had good sensitivity but higher false alarm rates [45].

Most of these studies have been conducted on patients outside of normal everyday movement. Noise associated with normal movement and occurring from the surrounding environment, has yet to be dealt with, so the use of accelerometry to detect motor seizures as a viable all in one solution is not yet an option. However, its use as part of a larger hybrid solution may be viable.

\section{Local field potentials}

There has been significant recent interest in the use of local field potentials (LFPs) as a biomarker for movement disorders such as Parkinson's disease and essential tremor [46-48]. Discussions have not been so prevalent in regards to closed loop systems for epilepsy, however, 
there is evidence to suggest that LFPs could be a source of biomarkers for seizure prediction and detection [49].

Aibel [49] used microelectrodes inserted at temporal lobe regions, with wide bandwidth, high frequency recorders $(0-6 \mathrm{kHz}, 30 \mathrm{ksamp} / \mathrm{s}, 10000$ gain) while evaluating six mesial temporal lobe epilepsy patients during seven seizures. The recordings showed that clear epileptiform discharges could be seen in the LFP between approximately $3 \mathrm{~s}$ after and $20 \mathrm{~s}$ prior to seizure onset. Regarding hypersynchronous onset pattern seizures with ictal discharges $<2 \mathrm{~Hz}$, prior to seizure onset fast ripples of increasing power occurred, they were also associated with an increase in the rate of ictal ripple and fast ripple, and individual spikes showed a ripple followed by a fast ripple. Although the sample size was small, the results were encouraging due to the similarity between seizures. Moreover, several animal models have recently been assessed to evaluate the potential of LFPs for seizure prediction and detection. Kainate-treated rats were used to evaluate the LFPs of the anterior nuclei of the thalamus (ANT) using the power efficient Generic OsorioFrel algorithm to predict clinical seizures in TLE. From a total of 161 segments of ictal data and 103 segments of interictal data, the algorithm using LFPs from the ANT was able to predict the seizure onset with $100 \%$ sensitivity and $94 \%$ sensitivity with a FPR of 0.5 per hour in real time before the clinical onset of the seizures, and was preceding the prediction by the ECoG in $75 \%$ of cases [50]. Recently a study had some success in detecting seizures in zebra fish using only single channel LFP recordings in genetic and chemical induced seizures using both invasive and non-invasive LFP recording techniques [51].

A majority of LFP seizure detection models have yet to be clinically tested. Animal models have shown results that LFPs have potential to be a viable and perhaps even more accurate alternative to EEG based prediction/detection models. The works of [52] and later [53] suggest that applying brief pulse stimulation within $2 \mathrm{~s}$ of the occurrence of neuronal after-discharges (AD), used as a model for epileptiform activity, has 4.5 times more chance of stopping the $\mathrm{AD}$. With this in mind, the latency values associated with LFPs could be useful to closed loop stimulation models.

\section{Video monitoring}

Video monitoring is part of the gold standard for detecting seizures. Although used on its own, at this point, they have had limited use due to the requirement of the patient to be in the area of the camera to be effective, although this may be less of an issue during the night [16].

Video monitoring can use motion path methods based on tracking the space/time trail of moving objects. Velocity, area, angular speed, oscillation or rotation are some of the other values used to detect seizures by finding and interpreting motor patterns in video footage $[14,15]$.

Video methods can be marker aided or marker free. Marker aided methods require objects worn on joints or extremities which can be uncomfortable for the patient. Research in automated video analysis is limited, while progression has been made in recognizing various kinematic patterns in epileptic seizures, studies have been limited to recognising a limited set of seizures. It would be beneficial for progression to be made in a video system with a holistic view of epileptic seizures [54].

\section{Other methods}

There are several other methods that have been studied for the prediction of epileptic seizures. A limited study consisted of inducing periodic electrical stimulation of cortex and assessing the intercranial EEG results using feature extraction and comparison in the interictal, preictal and postictal periods [55]. The study showed some interesting results that may result in further investigation, however, for the purpose of a closed loop neurostimulation device, the fact that it requires a constant although periodic stimulation may defeat the purpose of a closed loop system of this kind.

In 1997, [56] showed that there was a significant sustained increase in blood flow $10 \mathrm{~min}$ prior to a seizure in the epileptic temporal lobe, and at 2 min pre-ictus, an increase in both epileptic and non-epileptic temporal lobes. Another study discussed optical measurements of blood flow and oxygenation in animal and human neocortex. It concluded that the method could become important in predicting epileptic events [57]. The studies show that this method maybe of value in closed loop systems but maybe held back only by the availability and cost of wearable imaging devices.

Electromyography (EMG), a system that detects muscle signals, has been a subject of some studies that have resulted in wearable devices that have been on the market for some time. A study completed on one device showed a $91 \%$ sensitivity in detecting generalised tonicclonic seizures in 11 patients within an average of $20 \mathrm{~s}$ of seizure onset [58]. Other proposals have shown mixed results when tested on other seizure types such as clonic seizures [59]. Although it has been found that EMG can produce reasonable results for certain seizure types and in certain muscle groups, currently it requires a specific type of seizure with specific placement to be of use as a one stop detection system, however, it has potential as part of a multimodal system.

Perhaps an important method of seizure prediction that has had less of a mention in previous reviews than it deserved, is self-prediction of oncoming seizures. A study conducted by [60] in 1997 focused on a questionnaire given to a relatively large group (562) of epilepsy 
patients with both generalised or focal seizures with a large demographic range. Patients were asked questions regarding warning and initial symptoms of epileptic seizures (WISE). Results showed that at least $47 \%$ of patients had prior warning of oncoming seizures, and the clear majority of these had at least $10 \mathrm{~s}$ warning until seizure onset. Further to this, a majority of those patients were able to commit spontaneous actions or follow instructions [60,61]. found from a study of 49 patients that $67 \%$ experienced some form of aura before a seizure, and of these $85 \%$ could react during this time. However, [60] also found that there were also episodes where WISE occurred without a following seizure. This evidence shows there is potential for patient prediction of epileptic seizure, however, there is concerns that any seizure intervention method used maybe habit forming for the patient [60]. Therefore, there are ethical boundaries to cross, and the method may only be successful if used as part of a multimodal method.

Other methods include mattress detectors, seizure alert dogs, electrodermal activity and audio systems such as baby monitors. However, these methods are limited in use and would not be a focus for this application.

\section{Multimodal methods}

Using multimodal methods have the potential to improve sensitivity and reduce the number of FPs in detection systems. These methods include combinations of those previously discussed, and include the same processes of feature extraction and thresholding or training processes that potentially provide a higher degree of resolution.

A MATLAB based software called EPILAB was designed and distributed as a free software for the use of researchers to perform studies in epilepsy prediction and detection. It allows the use of multiple algorithms to predict seizures from EEG data. It can be user adapted to allow the process of ECG/EEG data for prediction or detection of seizures $[62,63]$.

A study using 12 recorded (simultaneous) EEG and ECG (HRV) neonatal data, using a concatenate statistical based classifier model for seizure detection, was conducted with encouraging results. An independent and patient specific model was tested resulting in a $97.52 \%$ sensitivity, and $13.8 \%$ FPR for the patient specific model, while the results of 81.44 and $28.57 \%$, respectively, were found for the independent model [64]. While this is encouraging, the dataset is limited to neonatal subjects, there is no mention of latency measurements and the greatest results were obtained in patient specific algorithms. In 2016, [65] developed an ECG/EEG method for seizure detection that was tested on 10 patients with diverse epilepsy symptoms from the EPILEPSIAE project [63]. It obtained $100 \%$ sensitivity and $99.91 \%$ specificity with an average latency of $2.6 \mathrm{~s}$. Although there was a FP rate of $3 / \mathrm{h}$, the method has the potential for online use, and testing on intercranial EEG data and a larger data base would be beneficial. Further testing could incorporate blood flow analysis or ACM data to lower False Positive rates and prediction analysis should be the focus of further studies.

Other methods proposed include combinations involving ACM, EMG or electrodermal methods to detect motor seizures. Although there is ongoing research into multimodal detection methods, there are a number of commercially available devices or devices that are still under testing that use a combination of these methods to detect seizures for patient warning systems [14]. However many of the commercial devices have no valid scientific data associated with them or are useful for only certain epileptic conditions [66].

Recently, a study conducted on a highly heterogeneous population of 69 epilepsy patients with nearly $6000 \mathrm{~h}$ of data, tested a model using a combination $22 \mathrm{ACM}$ and 3 electrodermal features collected from wrist worn sensors. While patients engaged in some normal everyday activities, they did not engage in sports or physical labour, so while further testing in that environment is required, sensitivities of nearly $95 \%$ with a FPR of only 0.2 per day were achieved. However, the results show a median latency of $29 \mathrm{~s}$ [67], its use in online applications may be minimal and certainly for any closed loop neural stimulation application.

As a final example, [68] presents data obtained from simulated seizures using healthy patients to test a multimodal system made up of EMG, ACM and gyroscope data. The sensor system was integrated into a single suit. Although the suit was uncomfortable, even for healthy patients, due to the large number of electrodes, it produced some promising results for average sensitivity and FPRs. It was tested during everyday activities using wavelet transforms for feature extraction [68]. Although average latency was generally $1 \mathrm{~s}$ or below, more testing on real patients would be required to ensure the relevancy of these results.

\section{Control of refractory epilepsy}

For those patients with refractory focal epilepsy that have either found resective surgery to be insufficient, impracticable or those that are fearful of the irreversible nature of these procedures, neurostimulation maybe a viable option to help control seizures. Moreover, as epilepsy is a chronic condition, it is encouraging that recent long term studies, even if all had some methodological problems, have shown most forms of invasive neurostimulation have had increasing efficacy over time with no adverse effects apart from device related complications [69]. 
An advantage of stimulation of focal epilepsies over AED systems that targets all areas of the brain stimulators are designed to target the area only where the seizure manifests [70]. However, the choice of stimulation methods will depend on whether the area of epileptic focus is well defined, or if it has several focal areas or extended regions, and where it is located. The patient's tolerability is also an area a clinician must account for when choosing an appropriate stimulation method.

Table 1 compares an outline of three approved invasive stimulation devices for the treatment of epilepsy, a brief description of each stimulation method, and a review of its side effects.

\section{Vagal nerve stimulation}

Vagal nerve stimulation has been approved by the US Food and Drug Administration (FDA) and in Europe since 1997 [70, 71]. The procedure involves implanting a pulse generator into the upper left side of the chest, and a lead is threaded to stimulate the left vague nerve at regular intervals by attaching the positive and negative electrodes to the nerve via a tethering anchor [72]. The procedure can be completed as either an inpatient or outpatient with either a general or local anaesthetic. The pulse generator is activated approximately 2 weeks after the procedure where the pulse is set at patient specific currents, frequencies and durations. In general terms these parameters are in the vicinity of: $1.0-2.0 \mathrm{~mA}$, $500 \mu$ s pulse width, $20-30 \mathrm{~Hz}, 30 \mathrm{~s}$ ON, and $5 \mathrm{~min}$ OFF.

The maximum current is reached gradually using 0.25 $\mathrm{mA}$ increases. Moreover, patients are given a magnet that permits them to stop stimulation in case of complications or certain events, by positioning it over the pulse generator. Alternatively, they can deliver a single stimulation using pre-programmed parameters by passing the magnet over the battery $[71,72]$.

Although the exact mechanisms of VNS are not known, it is known that during VNS the unmyelinated type $\mathrm{C}$ fibers of the vagas nerve are stimulated. As described by [72], the device components are available as a kit and a sample of one such kit includes: (i) single pin Titanium-housed pulse generator with a Li/CFx battery (life of 6-8 yrs.), (ii) a $43 \mathrm{~cm}$ lead wire with two platinum/iridium helical electrodes and a helical tethering anchor, and.

(iii) a disposable subcutaneous tunnelizer. Most pulse generators today detect the heart rate for use in as a closed loop system [72].

In a retrospective study of 100 patients, [73] found that, on average, a half of patients found a $50 \%$ reduction, while a quarter found a $75 \%$ reduction in seizure. These findings were comparable with previously conducted studies. It must be noted that some patients found no change or worsening seizure rates following the procedure. Moreover, median seizure reduction trended upwardly over a 12 year period and although $50 \%$ of patients found side effects from the procedure, only 1 patient from 100 had side effects so intolerable, the device required removal [73]. Stimulation adverse effects include voice alteration, cough, dyspnoea, paraesthesia, headache and pain [74]. Patients receiving the treatment usually report improvement in mood, memory, cognition and general quality of life [72].

\section{Thalamic stimulation}

There have been several studies conducted on intracranial stimulation with a focus on managing epileptic seizures. Some of the network areas focused on are: the cerebellum, subthalamic nucleus, centro median thalamic nuclei,

Table 1 Approved invasive stimulation methods for epilepsy. Replicated from [70]

\begin{tabular}{|c|c|c|c|}
\hline Data & Vagus nerve stimulation & Thalamic stimulation & Responsive focus stimulation \\
\hline Approval & $\begin{array}{l}1997 \text { (FDA)/(EU) (also heart rate triggered closed } \\
\text { loop since 2015) }\end{array}$ & $2011(E U)$ & $2013(F D A)$ \\
\hline $\begin{array}{l}\text { Stimulation } \\
\text { site }\end{array}$ & Left vagus nerve (neck) & $\begin{array}{l}\text { Anterior nuclei of the thalamus } \\
\text { (bilaterally) }\end{array}$ & Epileptic focus (cortex) \\
\hline $\begin{array}{l}\text { Stimulator } \\
\text { placement }\end{array}$ & Subcutaneous, left pectoral/sub clavicular & Subcutaneous, abdominal & Within the skull \\
\hline $\begin{array}{l}\text { Stimulation } \\
\text { mode }\end{array}$ & $\begin{array}{l}\text { Open-loop/closed-loop based on detection } \\
\text { of tachycardia }\end{array}$ & Open-loop & $\begin{array}{l}\text { Closed-loop based on detection of } \\
\text { ictal EEG patterns }\end{array}$ \\
\hline $\begin{array}{l}\text { Stimulus } \\
\text { parameters }\end{array}$ & $\begin{array}{l}\text { Intensity: } 0.25-3 \text { mA Frequency: } 20-30 \mathrm{~Hz} \\
\text { Pulse width: } 250-500 \mu \text { s Duty cycle: } 30 \text { s on/ } 5 \text { min } \\
\text { off (standard); } \\
7 \text { s on/30 s off ("rapid cycling") }\end{array}$ & $\begin{array}{l}\text { Intensity: } 5 \mathrm{~V} \\
\text { Frequency: } 145 \mathrm{~Hz} \text { Pulse width: } 95 \mu \mathrm{s} \\
\text { Duty cycle: } 1 \mathrm{~min} \text { on/5 min off }\end{array}$ & $\begin{array}{l}\text { Intensity: 1 mA Frequency: } 200 \mathrm{~Hz} \\
\text { Pulse width: } 160 \text { Hs Duty cycle: } \sim 5.9 \\
\text { min/day; (closed loop) }\end{array}$ \\
\hline $\begin{array}{l}\text { Side effects } \\
\text { of implantation }\end{array}$ & $1.6 \%$ infections $1 \%$ vocal cord paralysis & $\begin{array}{l}12,7 \% \text { infections } 10.9 \% \text { local pain } 18.2 \% \\
\text { paraesthesia at implantation site } 4.5 \% \\
\text { in cranial bleeding }\end{array}$ & $\begin{array}{l}7.8 \% \text { infections } 4.7 \% \text { intracranial } \\
\text { bleeding }\end{array}$ \\
\hline $\begin{array}{l}\text { Side effects of } \\
\text { stimulation }\end{array}$ & $\begin{array}{l}\text { Hoarseness (intensity-dependent up to } 66 \% \text { ) } \\
\text { Cough (up to } 45 \% \text { ) }\end{array}$ & $\begin{array}{l}14.8 \% \text { depression } 13.0 \% \text { memory } \\
\text { impairment }\end{array}$ & No statistics \\
\hline
\end{tabular}


caudate nucleus and hippocampus. Each of these areas have shown effectiveness in specific types of epilepsy [75]. While some of these studies have had results that are encouraging, enough to justify future larger studies, the bilateral deep brain stimulation of the anterior nuclei of the thalamus (ANT-DBS) is the only area, apart from the epileptic foci, that has gained widespread approval for stimulation [70, 76].

Like VNS, the exact mechanisms of action of stimulations are largely unknown. In a recent article, [77] suggested that high frequency ANT-DBS "may override the neural circuitry by blocking pathological activity and replacing efferent output". There are several other suggestions for mechanisms of action and it is still a debated topic [77]. It has been shown previously, however, that high frequency stimulation can block epileptic activity in cerebral cortex while low frequency of below $1 \mathrm{~Hz}$ tends to synchronise activity [78].

Halpern [79] describes the implantation of the system by using an internal deep brain stimulation (DBS) pulse generator, generally placed in a sub clavicular pocket, bilaterally. The stimulation leads used during surgery are "DBS depth electrodes with 4 platinum-iridium stimulation contacts $1.5 \mathrm{~mm}$ wide, with $1.5 \mathrm{~mm}$ edge-to-edge separation because the ANT is relatively larger than other DBS targets" [79]. The lead is then secured to a burr-hole cap. A contralateral electrode is similarly inserted. Finally, an extension wire is tunnelled subcutaneously and attached to the leads and the implantable pulse generator [79]. Currently, a typical commercially available kit contains: (i) dual-channel implantable pulse generator kit and $7200 \mathrm{mAh}$ battery (life of 5.8-10.5 years) including a magnetic switch, remote wireless programming, frequency programmable on each channel with up to 13 stimulation program storage, (ii) implantable lead kit for DBS, $1.27 \mathrm{~mm}$ diameter lead with 4 contacts, $1.5 \mathrm{~mm}$ spacing and length, $300 \mathrm{~mm}$ or $400 \mathrm{~mm}$ lead length, (iii) implantable extension kit for DBS, length $52 \mathrm{~cm}$ Includes anchorage and tunnelling tools, (iv) clinician programmer, (v) external pulse generatorfor testing during surgical procedure, (vi) patient Programmer- for patient to change stimulation program as required and check battery life, and (vii) wireless remote programmer for clinician [80].

European approval for the procedure was granted following the randomized double-blind controlled trial of stimulation of the anterior nuclei of thalamus for epilepsy (SANTE) in 2010. The trial was extended to 5 years and involved 110 patients culminated with the release of results in 2015. The evidence presented was of class 1 , however, there was a widespread variety of AEDs used during the trial. The trial showed that the procedure had a median seizure reduction of $41 \%$ at year 1 , and $69 \%$ at year 5 . Moreover, the responder rate, which is defined as a patient with a greater than $50 \%$ reduction in seizures, was $43 \%$ at year 1 and $68 \%$ at 5 years and the QOL in Epilepsy measure also showed significant improvement over the 1 and 5 -year periods. There were minimal adverse effects over the trial period [81]. Recently, following the release of the 7 year data in 2017, the FDA approved the use of Medtronics ANT DBS System for use in the US [82]. Furthermore, there have been a number of other studies conducted that have given comparable results to these studies [83].

DBS of well-defined anatomic targets especially ANTDBS has been shown to be effective in the short term and with greater efficacy over the longer term. To be effective they should be high frequency pulses of $5 \mathrm{~V}$ intensity although this can be a matter of patient specificity.

\section{Focal area stimulation}

RNS is a system given FDA approval in 2013, after the results of a trial conducted in 2011. The RNS is designed for sufferers of focal epilepsy. It involves the electrical stimulation of up to two epileptic focus areas conducted by either depth electrodes or subdural cortical strip leads. The electrical stimulation is given in response to EEG patterns before ictal onset [83]. The intermittent stimulation is delivered to the epileptic focus area/s with the intention of limiting or inhibiting any abnormal electrical activity and preventing it from becoming clinical seizure [84]. Although this implies a closed loop system only, the mechanisms and results of the stimulation is discussed in this section. Unlike the ANT-DBS system the stimulator system is fully implanted in the skull. The neurostimulator is implanted in a burr hole in the patient's skull and is connected to the electrodes via thin flexible wires. The electrodes are placed at the seizure onset zones, either bilaterally or at two onset zones depending on epilepsy type and can be placed in either deep structures (e.G. hippocampus) of the brain or on the surface. The four contacts on each of the electrodes can be predetermined as either anode or cathode, the stimulator case can serve as a cathode if required [84].

The electrical stimulation applied is biphasic, current controlled with high frequency. Typical stimulation parameters are between 1 and $3 \mathrm{~mA}$, pulse width of $160 \mu \mathrm{s}$, pulse duration between 100 and $200 \mathrm{~ms}$, and a frequency ranging between 100 and $200 \mathrm{~Hz}$. It is noted that stimulation quantity is controlled by detection, however, compared with DBS, the total current density delivered remains low $[70,84]$. The original works in this area, leading to this method, was conducted by [52]. The resulting work conducted on after discharges (AD) showed that the most effective stimulation was brief, between 0.5 and $1 \mathrm{~s}$ and needed to be applied within $4.5 \mathrm{~s}$ of the $\mathrm{AD}$ to abort it and similar bursts maybe effective on epileptogenic forms [53]. Controlled shorter-term 
studies, and uncontrolled longer-term studies, showed improved efficacy over time and improve QOL [85-88]. The results of the stimulation, however, almost certainly, have some connection to the system's closed loop structure, so will not be discussed in this section.

\section{Non-invasive stimulation}

Transcranial stimulation techniques such as transcranial magnetic stimulation (TMS) and transcranial direct current stimulation (tDCS) are techniques that can modulate cortex excitability and activity without the need for an invasive procedure. Moreover, other noninvasive techniques involving external stimulation of the vagas nerve or the trigeminal nerve are other areas that could deliver results in the control of epileptic seizures.

\section{Transcranial magnetic stimulation}

TMS is a method of focal brain stimulation first described in 1985, where strong fluctuation magnetic fields created externally to the brain induce internal electric currents. TMS has several protocols that distinguish between pulse repeating length. In particular, low frequency $(<1 \mathrm{~Hz})$ repetitive TMS (rTMS) has been tested as a means of reducing cortical excitability. Like most methods of stimulation for epilepsy, TMS has been found to be patient specific. Parameters such as frequency, intensity, type of stimulation coil, area of application, duration of stimulation, and the interval between trains have shown to differ significantly between patients $[89,90]$. Risks associated with the use of this method have been found to be minimal, however, testing of the effectiveness of the treatment has produced mixed results, ranging from very, to not at all effective [89-91]. This may be due to the great variety in parameters used, and while the effectiveness is largely unknown, the noninvasive nature of the treatment warrants further investigation in controlled trials.

\section{Transcranial direct current stimulation}

tDCS is a non-invasive method of stimulation that requires cortical direct current (DC), polarity dependent, stimulation. The stimulation has been shown to reduce or increase cortical excitability, dependent on polarity, for up to an hour after stimulation. The addition of certain medication can also help to prolong these effects [90]. To reduce seizures in epileptic patients, the general method involves placing sodium chloride solutionsoaked sponge electrodes in specific positions on the head. The cathode is placed directly over the epileptogenic focal zone and the anode is placed over an area without epileptogenic activity contralateral to the stimulated side. Then a specific quantity of current at a certain frequency for a specified amount of time is applied. The procedure has generally been shown to be safe, well tolerated by the patient and relatively easy to apply [92]. Similar to TMS, clinical trials of the method have been limited, of mixed results, and have generally used different stimulation patterns [92]. A recent, small, sham controlled study conducted in 2017 showed a reduction in seizure activity [93], and shows that while trials of the method are in its infancy, there is evidence to suggest that larger controlled trials are required for what could be a beneficial, non-invasive therapy.

\section{Transcutaneous Vagus nerve stimulation}

Recently, interest has progressed in a non-invasive method for vagal nerve stimulation. A small area of skin near the ear is innervated by the vagus nerve, and gentle stimulation has been shown to produce similar results in terms of fMRI brain activity changes as the surgically implanted device [94]. The method is generally patient specific, with changeable settings for frequency and current, with trials being conducted between 20 and 25 $\mathrm{Hz}$, and approximately $1 \mathrm{~mA}$. The length of time these currents are applied, and frequency of application are other parameters that can be disputed [95, 96]. This method of stimulation has been approved in Europe and the US for the treatment of a diverse range of conditions including one device for the control of epilepsy [74].

In the past, testing on epilepsy has had mixed results of efficacy and this again could be the result of the patient specificity of the method. However, results shown in studies such as the pilot study conducted on paediatric patients in China, and the double-blind randomised study conducted in Germany show encouraging results and good patient tolerability $[95,96]$. These studies, coupled with the recent studies showing the increased efficacy with time of the surgical vagal nerve stimulation method, gives good cause for the need of future long-term controlled trials of tVNS for the control of epilepsy.

\section{External trigeminal stimulation}

An alternative to tVNS is external trigeminal stimulation (eTNS). This form of stimulation is a relatively new therapeutic technique involving the stimulation of the trigeminal nerve. The trigeminal is the fifth cranial nerve [97], and like the previous methods, the exact mechanisms of action are relatively unknown.

Trials of eTENS for the control of epilepsy have generally involved using an external pulse generator connected to two electrodes placed above the eyebrows. In a 2013 trial [98] describes placement of electrodes that were "specifically designed to contact the right and left branches of the ophthalmic and supratrochlear nerves to provide bilateral stimulation". Measured parameters used in trials were frequency, current, pulse width, with varying stimulation length times [98-100]. The frequency with greatest results in animal tests were $>100$ 
$\mathrm{Hz}$ and so this was adopted in human trials, currents used varied but generally $<20 \mathrm{~mA}$ was used.

Studies that were conducted include a 6 month, 7 patient open proof of concept trial [99], an 18 week 50 subject double-blind randomized active-control trial [98] among others. Although results were mixed, they showed that the treatment was generally well tolerated, with little side effects relative to invasive methods, and they showed a general trend to improved efficacy over the time-period with an increase in mood and quality of life indicators [98-100]. The results show that larger multicentre controlled trials of eTENS would be advantageous for the pursuit of a non-invasive solution. Moreover, these trials have had similar parameter settings between patients, but due to the sometimes-large difference in beneficial parameter settings between patients, it may be advantageous to also take a patient specific approach during future testing.

\section{Closed loop neurostimulation}

Systems involving DBS or other electrical based stimulation for the control of diseases such as Parkinson's, essential tremor, depression among others have been found to be an acceptable form of treatment. Many of these systems use an open loop form of stimulation to continually induce stimulation to control what can be ongoing effects of the disease. However, epilepsy ictal periods occur after relatively long periods of interictal periods [101]. Although continual use of neural stimulation has shown to have minimal long-term side effects, other factors such as battery power and device wear could become problems with open loop systems due to continual use. In addition, other benefits could include early warning systems for prevention, by careers or patients, of secondary injury or sudden unexplained death in epilepsy patients. The ability to adjust levels of stimulation automatically if the current stimulation parameters are insufficient could also be beneficial [102]. Moreover, a permanent seizure detection or prediction sensor that records ongoing data could be vital for research, and further understanding of the disease and development of treatments. Currently, there are several commercially available devices available, and several suggested or trialled closed loop systems.

\section{Closed loop vagal nerve stimulation}

In 2014, Cyberonics obtained CE mark and US FDA approved for use a vagal nerve stimulation therapy that utilises a closed loop invasive system based around the Aspire SR implantable generator. This technology was again upgraded in 2017 with the approval of the SenTiva model [103]. The therapy is a device that builds on the open loop VNS system discussed in the previous section. While the original system had some closed loop functionality in the form of a magnetic switch that activates stimulation at the behest of the patient or patients caregiver [102], the second generation pulse generator incorporates a sensor that implements a cardiac based seizure detection algorithm. Greater than $80 \%$ of epileptic seizures result in ictal tachycardia $[36,104]$ which is the basis of this system. The system can be useful for patients that have shown at least a $20 \%$ increase in heart rate and can be set to trigger using a predetermined threshold value to minimise the FPs stimulated by the system $[104,105]$.

A multicentre trial of the Aspire SR model involving 31 patients over 12 months showed the effectiveness of the cardiac based seizure detection algorithm and was highly patient and seizure specific. However, of those seizures that were detected, they were close to or even before ictal onset. Moreover, the trial showed that the effectiveness of the stimulation at the time of a detected seizure, could possibly reduce the severity of the seizure significantly [104].

A recently completed longer term study of 113 patients, with the Aspire SR system, had more encouraging results. The study involved patients under the care of one surgeon who had received new insertions of the model (51 patients) and those that had old VNS models that were updated at battery change (62 patients). The new (59\% at a median of 13 months after insertion) and changed model patients both had better than expected responder rates over the period including a high rate of patients experiencing an additional seizure reduction in the changed model patients $(71 \%$ had $>50 \%$ reduction in seizures following battery change) [106].

These results are mixed and somewhat complementary, however, this is most likely be due to technical difficulties in establishing the correct thresholds for the cardiac based detection systems and could depend highly on patient specific or seizure specific symptoms. What is encouraging, is the noted significant reduction in seizure severity when stimulation is applied before or during a seizure. This is a potential advantage that closed loop systems can bring to seizure control when opposed to open loop control. Open loop stimulations distribute stimulations repetitively for a pre-set duration and may not necessarily be applied during an ictal period to attempt to disrupt spontaneous epileptiform discharges.

\section{Responsive neural stimulation}

There have been many small uncontrolled clinical and animal studies conducted on responsive stimulation for epilepsy over the last two decades. The studies have included targeting several different areas such as ANT, thalamic, cortical and epileptogenic zones with mixed results [107], however [108] is the only study in this area to produce class 1 results focusing on the epileptogenic 
zone. This study showed a mean seizure reduction of $37 \%$ during the 3-month follow up period, the results lead to the approvals for such a device in Europe and the US in 2013. This approval was gained for the commercially available RNS system designed and manufactured by NeuroPace. The stimulation system, described in a previous section, delivers pre-programmed electrical stimulation to up to 2 epileptogenic focus areas to attempt abortion of spontaneous epileptiform discharges. The key to success of this type of seizure prediction is in choosing the correct target area [108]. The depth electrodes or subdural cortical strip leads also act as ECoG recording instruments. The neurostimulator provides stimulation to the seizure focus once a seizure is detected. The system also provides wireless connection with the stimulator device for clinical programming and extraction of patient clinical data that can be uploaded to a database easily by the patient or carer [109].

The algorithms used for detection, utilises a choice of three tools, line length, bandpass and area to interpret the ECoG data [102]. The features are extracted, and the recent window of data is compared to the long-term trend, when the recent window trend is greater than the long-term trend by a predetermined percentage the stimulus is executed. The tool or tools used are patient specific in that they are utilised by how seizures typically manifest in terms of their ECoG recordings in a particular patient [107]. The tools are optimised so that real time detection requiring low computational power can be achieved within the constraint of the implantable stimulation device [102]. Once the pre-programmed stimulation pulse-train is delivered, the detection algorithm checks to see if the activity remains present and if so, up to an additional four therapies consisting of up to 2 pulse trains can be delivered, each of these can be of varying parameters [107]. A 7-year long term treatment study of 111 patients with mesial temporal lobe epilepsy that had undergone the RNS treatment showed, using a last observation carried forward method, a 70\% reduction in seizures with $29 \%$ having seizure free periods greater that 6 months, the treatment was well tolerated by patients [110]. These results show good long-term efficacy for RNS treatment and are comparable to other long-term efficacy data for open loop.

\section{Other closed loop systems}

Several closed loop systems have been tested both on animals and humans over the last two decades to find an appropriate system to initiate the various types of stimulation methods following real time detection of an identified seizure or epileptiform discharge.

These systems have both been invasive and non-invasive in nature, or a combination of both, and several recent attempts are interesting. In 2012, a team demonstrated a closed loop system using TES on rats that could be effective [101]. Studies conducted on the on-demand mode of VNS systems, where the VNS is initiated by patients or caregivers showed between 53 and $66 \%$ of patients reported being able to interrupt seizures themselves [111]. To improve on this, a clinical trial was performed that utilised the Digitrace 1800 Plus from SleepMed Inc. to collect EEG and ECG data. The system used the data from 5 patients with patient-specific algorithms to classify seizure or epileptiform discharges, activating an electromagnet positioned over the implanted generator to initiate VNS as required. The EEG or ECG data was used unconnected to distinguish patterns and initiate VNS. While the results showed good sensitivity, specificity and a short latency, it was suggested that fusing the datasets and even adding movement data such as accelerometry to the algorithm may produce a more sophisticated, accurate result [111].

\section{Discussion and future studies}

Currently, there are several available systems that enable neurostimulation with a feature to control epilepsy for those patients whose options are limited. These devices are effective for a significant portion of seizures; however, the VNS closed loop versions yield similar results to open loop versions. Considering the studies described in this review, the large improvement in responder rates involving patients that received an upgrade to closed loop systems from their current system, there may be room to improve the VNS system by improving the detection algorithm.

Studies of the RNS system discussed in this review have not presented data indicating the effectiveness of the detection system used in the system. In the original works, the detection algorithms, described in [102], have the distinct advantage of being power conservative. They showed results in their development studies [112-114] that may now be inferior when comparing them to some of the newer studies presented in this review. With technological advances in processing ability, it could be suggested that there may now be room to improve on existing systems. According to ref. [108], the risks associated with the system's use are comparative when taken in context with the risks associated with anti-epileptic drugs and are mainly associated with risks associated with any surgical implantation procedure. However, the benefits of having a full-time system recording brain activity similar to RNS are many. On an individual basis they potentially allow practitioners to make informed decisions of the patient's progress and diagnose any changes in the epileptiform discharges. In a broader sense it can give researchers valuable data they have lacked in the past to possibly help better understand the condition and aid in design of more effective systems. Table 2 gives a description of the currently approved 
Table 2 Approved open and closed loop stimulation devices for the treatment of epilepsy and reported efficacy

\begin{tabular}{|c|c|c|c|c|}
\hline Data & Vagus nerve stimulation & Vagus nerve stimulation & Thalamic stimulation & Responsive focus stimulation \\
\hline Stimulation site & Left vagus nerve (neck) & Left vagus nerve (neck) & $\begin{array}{l}\text { Anterior nuclei of the } \\
\text { thalamus (bilaterally) }\end{array}$ & Epileptic focus (cortex) \\
\hline Stimulator placement & $\begin{array}{l}\text { Subcutaneous, left } \\
\text { pectoral/sub clavicular }\end{array}$ & $\begin{array}{l}\text { Subcutaneous, left pectoral/ } \\
\text { sub clavicular }\end{array}$ & Subcutaneous, abdominal & Within the skull \\
\hline Stimulation mode & $\begin{array}{l}\text { Open-loop } \\
5 \mathrm{~m} \text { off } 30 \mathrm{~s} \text { on }\end{array}$ & $\begin{array}{l}\text { Closed-loop based on } \\
\text { detection of tachycardia }\end{array}$ & $\begin{array}{l}\text { Open-loop } 5 \text { m on } 1 \text { m } \\
\text { off }\end{array}$ & $\begin{array}{l}\text { Closed-loop based on detection } \\
\text { of ictal EEG patterns }\end{array}$ \\
\hline Seizure reduction & $\begin{array}{l}b_{30.43 \%} \text { at } 12 \text { months } \\
62.68 \% \text { at } 6 \text { years [73] }\end{array}$ & ${ }^{\mathrm{a}} 66 \%$ at 13 months [106] & $\begin{array}{l}{ }^{c} 41 \% \text { at year } 1 \\
69 \% \text { at year } 5[81]\end{array}$ & $\begin{array}{l}60 \% \text { year } 3 \\
66 \% \text { year } 6[87]\end{array}$ \\
\hline Responder rate & $\begin{array}{l}\mathrm{b}_{49 \%} \text { at time of last } \\
\text { follow up }\end{array}$ & a $59 \%$ at 13 months [106] & $\begin{array}{l}{ }^{c} 43 \% \text { at year } 1 \\
68 \% \text { at year } 5[81]\end{array}$ & $\begin{array}{l}58 \% \text { year } 3 \\
59 \% \text { year } 6[87]\end{array}$ \\
\hline $\begin{array}{l}\text { Effectiveness of detection/ } \\
\text { prediction algorithm }\end{array}$ & None used & No results have been given & None used & No results have been given \\
\hline
\end{tabular}

${ }^{a}$ Values are reported as mean values of the total new implant cohort

${ }^{\mathrm{b}}$ Mean length of treatment time was 4.10 years with a maximum treatment time of 12 years

'Values reported are median values of 110 patients

methods of stimulation available for epilepsy control, and gives an example of efficacy statistics that have been found in some studies. Furthermore, if applicable, it outlines the effectiveness of the detection algorithm employed in the system.

The review on potential prediction and detection methods shows that progress has been made in EEG methods although there is a trade off with computational power required for more accurate predictions, and the ability to produce online results is questionable. Moreover, the non-invasive requirements could promote social stigma for the patient as well as comfort and.

practicality issues in using the equipment. Certain ECG and other reviewed methods lack accuracy, but are promising, such as HRV which produces a more sophisticated detection and prediction than a simple assessment based on heart rate thresholding.

Alternatively, the work involving LFPs is a relatively new and seems to be promising with animal models, and larger scale tests should be considered as an alternative to ECoG methods due to its less computational power requirement. However, the greatest leverage in these systems involves using hybrid methods of detection/prediction. Combining two or more carefully chosen methods from ECoG, ECG, LFPs, accelerometry and other methods has the potential to improve prediction/detection of seizures that contribute to a more potent closed loop system.

In terms of stimulation methods, they may potentially be under-performing. Open loop methods administer stimulation in repetitive doses not necessarily at the seizure manifestation. There is some evidence to suggest that issuing VNS at the commencement or during a seizure can stop or at least reduce the seizure. Focal stimulation is based on studies that indicate stimulation at the epileptogenic source can stop seizure manifestation. To fully evaluate its effectiveness, a system that can guide the stimulation to occur directly before and during a seizure $100 \%$ of the time is required. The same could be said for ANT stimulation and other regional stimulation systems under investigation.

\section{Conclusion}

In conclusion, the ability to administer closed loop stimulation at seizure manifestation in a reliable and accurate way could potentially have a large effect on an epileptic patient, and their family's quality of life. It has the potential to fill the gap for the $30 \%$ of 50 million epilepsy sufferers that are drug-resistant and have found no respite either in new AEDs or from surgical attempts to relieve seizures. Moreover, continuous recording of biomarkers and seizure activity can potentially help researchers with important clinical information that has not been available to them by traditional reporting and tracking methods. Current systems show they are quite effective for a large portion of patients by reducing seizure activity, however, the results show they are not yet at the level of sophistication and effectiveness to eradicate seizures in all patients.

\section{Abbreviations}

ACM: Accelerometer; AD: After discharges; AED: Anti-epileptic drug; ANT: Anterior nuclei of the thalamus; ANT-DBS: Bilateral deep brain stimulation of the anterior nuclei of the thalamus; CSI: Cardiac sympathetic index; DBS: Deep brain stimulation; DC: Direct current;

ECoG: Electrocorticography; EEG: Electroencephalogram;

EMG: Electromyography; eTNS: External trigeminal stimulation; FDA: US Food and Drug Administration; FP: False positives; FPGA: Field-programmable gate array; FPR: False positive rate; HMM: Hidden Markov model; HRV: Heart rate variability; iEEG: Intercranial EEG; ILAP: International League Against Epilepsy; KNN: K-nearest neighbour; LFPs: Local field potentials; MOD: Model based matched wavelet; MSPC: Multivariate statistical process control; PCA: Principal component analysis; PPV: Positive prediction value; QOL: Quality of life; RLS: Recursive least squares; RNS: Responsive neuro stimulation; SANTE: Anterior nuclei of thalamus for epilepsy; SoC: System on chip; SRV: Support vector machines; tDCS: Transcranial direct current stimulation; TLE: Temporal lobe epilepsy; TMS: Transcranial magnetic stimulation; VNS: Vagal nerve stimulation; WISE: Warning and initial symptoms of epileptic seizures 


\section{Acknowledgements}

Not applicable.

\section{Authors' contributions}

$\mathrm{MDB}$ and $\mathrm{AZK}$ both equally contributed to the idea to review closed loop neural stimulation systems for refractory epilepsy. MDB conducted the majority of data collection and writing of the manuscript. AZK provided edits and revision. Both MDB and AZK approved the final manuscript.

\section{Funding}

Not applicable.

\section{Availability of data and materials} Not applicable.

\section{Ethics approval and consent to participate}

Not applicable.

\section{Consent for publication}

Not applicable.

\section{Competing interests}

The authors declare that they have no competing interests.

Received: 13 March 2019 Accepted: 10 October 2019

Published online: 29 October 2019

\section{References}

1. WHO WHO. Epilepsy. 2018

2. Laxer KD, Trinka E, Hirsch LJ, Cendes F, Langfitt J, Delanty N, et al. The consequences of refractory epilepsy and its treatment. Epilepsy Behav. 2014; 37:59-70.

3. J-t L, Liu B, Zhang H. Surgical versus medical treatment of drug-resistant epilepsy: a systematic review and meta-analysis. Epilepsy Behav. 2018;82 179-88.

4. Abramovici S, Bagić A. Chapter 10 - Epidemiology of epilepsy. In: Aminoff MJ, Boller F, Swaab DF, editors. Handb Clin Neurol. 2016;138:159-71.

5. Stafstrom CE, Carmant L. Seizures and epilepsy: an overview for neuroscientists. Cold Spring Harb Perspect Med. 2015;5(6):a022426.

6. NINDS NIoNDaS. The Epilepsies and Seizures: Hope Through Research 2017 [Available from: https://www.ninds.nih.gov/Disorders/Patient-CaregiverEducation/Hope-Through-Research/Epilepsies-and-Seizures-HopeThrough\#3109_13.

7. RS-k C, CYW L, CCA H, Yung A. Classifications of seizures and epilepsies, where are we? - a brief historical review and update. J Formos Med Assoc. 2017;116(10):736-41

8. Fisher RS, Acevedo C, Arzimanoglou A, Bogacz A, Cross JH, Elger CE, et al. ILAE official report: a practical clinical definition of epilepsy. Epilepsia. 2014; 55(4):475-82.

9. WHO WHO. Global Health Estimates 2015 Summary Tables: GLOBAL DALYs BY CAUSE, AGE AND SEX, 2000-2015 2016 [cited 2018 20th June]. Available from: http://www.who.int/healthinfo/global_burden_disease/estimates/en/ index1.html.

10. Aidan N, BG S, SS D, SJ W. Temporal trends in the mortality of people with epilepsy: a review. Epilepsia. 2010;51(11):2241-6.

11. Kwan P, Arzimanoglou A, Berg AT, Brodie MJ, Allen HW, Mathern G, et al Definition of drug resistant epilepsy: consensus proposal by the ad hoc task force of the ILAE commission on therapeutic strategies. Epilepsia. 2010;51(6): 1069-77.

12. Holmes G, Sirven J, Fisher RS. Temporal Lobe Epilepsy (TLE): Epilepsy Foundation; 2013 [cited 2018 20th June]. Available from: https:/www.epilepsy. com/learn/types-epilepsy-syndromes/temporal-lobe-epilepsy-aka-tle.

13. Koepp MJ. The help of biomarkers in the prevention of epilepsy. Lancet Neurol. 2016;15(8):782-4.

14. Ramgopal S, Thome-Souza S, Jackson M, Kadish NE, Sánchez Fernández I, Klehm J, et al. Seizure detection, seizure prediction, and closed-loop warning systems in epilepsy. Epilepsy Behav. 2014;37:291-307.

15. Ulate-Campos A, Coughlin F, Gaínza-Lein M, Fernández IS, Pearl PL, Loddenkemper T. Automated seizure detection systems and their effectiveness for each type of seizure. Seizure. 2016;40:88-101.
16. Van de Vel A, Cuppens K, Bonroy B, Milosevic M, Jansen K, Van Huffel S, et al. Non-EEG seizure detection systems and potential SUDEP prevention: state of the art: review and update. Seizure. 2016;41:141-53.

17. Alotaiby TN, Alshebeili SA, Alshawi T, Ahmad I, Abd El-Samie FE. EEG seizure detection and prediction algorithms: a survey. EURASIP J Adv Signal Process. 2014:2014(1):183.

18. Cook MJ, O'Brien TJ, Berkovic SF, Murphy M, Morokoff A, Fabinyi G, et al. Prediction of seizure likelihood with a long-term, implanted seizure advisory system in patients with drug-resistant epilepsy: a first-in-man study. Lancet Neurol. 2013;12(6):563-71.

19. Parvez MZ, Paul M. Prediction and Detection of Epileptic Seizure by Analysing EEG Signals; 2015.

20. Yoo J, Yan L, El-Damak D, Altaf MAB, Shoeb AH, Chandrakasan AP. An 8channel scalable EEG acquisition SoC with patient-specific seizure classification and recording processor. IEEE J Solid State Circuits. 2013:48(1):214-28.

21. Tessy E, Shanir PPM, Manafuddin S. Time domain analysis of epileptic EEG for seizure detection. 2016 International Conference on Next Generation Intelligent Systems (ICNGIS); 2016.

22. Faust $\mathrm{O}$, Acharya UR, Adeli $\mathrm{H}$, Adeli A. Wavelet-based EEG processing for computer-aided seizure detection and epilepsy diagnosis. Seizure. 2015;26: 56-64.

23. Sharma M, Bhurane AA, Rajendra AU. MMSFL-OWFB: a novel class of orthogonal wavelet filters for epileptic seizure detection. Knowl-Based Syst. 2018;160:265-77.

24. Zandi AS, Tafreshi R, Javidan M, Dumont GA. Predicting temporal lobe epileptic seizures based on zero-crossing interval analysis in scalp EEG. 2010 Annual International Conference of the IEEE Engineering in Medicine and Biology; 2010

25. Netoff T, Park Y, Parhi K, editors. Seizure prediction using cost-sensitive support vector machine. 2009 Annual International Conference of the IEEE Engineering in Medicine and Biology Society; 2009.

26. Behnam M, Pourghassem $\mathrm{H}$. Real-time seizure prediction using RLS filtering and interpolated histogram feature based on hybrid optimization algorithm of Bayesian classifier and hunting search. Comput Methods Prog Biomed. 2016;132:115-36.

27. Hooper S, Biegert E, Levy M, Pensock J, Spoel Lvd, Zhang X, et al., editors. On developing an FPGA based system for real time seizure prediction. 2017 51st Asilomar conference on signals, Systems, and Computers; 2017.

28. Alickovic E, Kevric J, Subasi A. Performance evaluation of empirical mode decomposition, discrete wavelet transform, and wavelet packed decomposition for automated epileptic seizure detection and prediction. Biomed Signal Process Control. 2018;39:94-102.

29. Smirnov Y, Popov A, Panichev O, Karplyuk Y, Kharytonov V, editors. Epileptic seizure prediction based on singular value decomposition of heart rate variability features. 2017 Signal Processing Symposium (SPSympo); 201712 14 Sept. 2017

30. Thuraisingham RA. Preprocessing RR interval time series for heart rate variability analysis and estimates of standard deviation of RR intervals. Comput Methods Prog Biomed. 2006;83(1):78-82.

31. Popov A, Panichev O, Karplyuk Y, Smirnov Y, Zaunseder S, Kharytonov V, editors. Heart beat-to-beat intervals classification for epileptic seizure prediction. 2017 Signal Processing Symposium (SPSympo); 2017.

32. Yamakawa T, Fujiwara K, Miyajima M, Abe E, Kano M, Ueda Y, editors. Realtime heart rate variability monitoring employing a wearable telemeter and a smartphone. Signal and Information Processing Association Annual Summit and Conference (APSIPA), 2014 Asia-Pacific; 2014

33. Fujiwara K, Miyajima M, Yamakawa T, Abe E, Suzuki Y, Sawada Y, et al. Epileptic seizure prediction based on multivariate statistical process control of heart rate variability features. IEEE Trans Biomed Eng. 2016;63(6):1321-32.

34. Moridani M, Farhadi $\mathrm{H}$. Heart rate variability as a biomarker for epilepsy seizure prediction; 2017. p. 3-8.

35. Jeppesen J, Beniczky S, Johansen P, Sidenius P, Fuglsang-Frederiksen A. Detection of epileptic seizures with a modified heart rate variability algorithm based on Lorenz plot. Seizure. 2015:24:1-7.

36. Fritz L, Christiana S, Stefanie L, Klaus P, Christoph B. Electrocardiographic changes at the onset of epileptic seizures. Epilepsia. 2003:44(3):348-54

37. Osorio I, Manly BFJ. Probability of detection of clinical seizures using heart rate changes. Seizure. 2015:30:120-3.

38. Cooman TD, Varon C, Vel AVd, Ceulemans B, Lagae L, Huffel SV, editors. Semi-supervised one-class transfer learning for heart rate based epileptic seizure detection. 2017 Computing in cardiology (CinC); 2017. 
39. Nijsen TME, Arends JBAM, Griep PAM, Cluitmans PJM. The potential value of three-dimensional accelerometry for detection of motor seizures in severe epilepsy. Epilepsy Behav. 2005;7(1):74-84.

40. Nijsen TME. Accelerometry based detection of epileptic seizures: Eindhoven University of Technology; 2008.

41. Gubbi J, Kusmakar S, Rao AS, Yan B, O'Brien T, Palaniswami M. Automatic detection and classification of convulsive psychogenic nonepileptic seizures using a wearable device. IEEE J Biomed Health Informatics. 2016;20(4):1061-72.

42. Borujeny GT, Yazdi M, Keshavarz-Haddad A, Borujeny AR. Detection of epileptic seizure using wireless sensor networks. J Med Signals Sensors. 2013;3(2):63-8.

43. Jallon P, Bonnet S, Antonakios M, Guillemaud R, editors. Detection system of motor epileptic seizures through motion analysis with 3D accelerometers. 2009 Annual International Conference of the IEEE Engineering in Medicine and Biology Society; 2009.

44. Villar JR, Menéndez M, de la Cal E, Sedano J, González VM. Identification of abnormal movements with 3D accelerometer sensors for seizure recognition. J Appl Log. 2017;24:54-61.

45. Jallon P, editor A Bayesian approach for epileptic seizures detection with 3D accelerometers sensors. 2010 Annual International Conference of the IEEE Engineering in Medicine and Biology; 2010.

46. Abosch A, Lanctin D, Onaran I, Eberly L, Spaniol M, Ince NF. Long-term recordings of local field potentials from implanted deep brain stimulation electrodes. Neurosurgery. 2012;71(4):804-14.

47. Christian MA, Gerd T, Marc HD, Hayriye C, Jean D, Peter B. Adaptive deep brain stimulation for movement disorders: the long road to clinical therapy. Mov Disord. 2017;32(6):810-9.

48. Shimamoto SA, Ryapolova-Webb ES, Ostrem JL, Galifianakis NB, Miller KJ, Starr PA. Subthalamic nucleus neurons are synchronized to primary motor cortex local field potentials in Parkinson's disease. J Neurosci. 2013;33(17): 7220-33.

49. Aibel WS, Catalina A-R, Anatol B, Eric B, Tony F, Itzhak F, et al. Ictal onset patterns of local field potentials, high frequency oscillations, and unit activity in human mesial temporal lobe epilepsy. Epilepsia. 2016;57(1):111-21.

50. An-chao $Y$, Da-wei $M$, Huan-guang L, Lin S, Kai $Z$, Hui Q, et al. The ability of anterior thalamic signals to predict seizures in temporal lobe epilepsy in kainate-treated rats. Epilepsia. 2016;57(9):1369-76.

51. Hunyadi B, Siekierska A, Sourbron J, Copmans D, de Witte PAM. Automated analysis of brain activity for seizure detection in zebrafish models of epilepsy. J Neurosci Methods. 2017;287:13-24.

52. Lesser RP, Kim SH, Beyderman L, Miglioretti DL, Webber WRS, Bare M, et al. Brief bursts of pulse stimulation terminate afterdischarges caused by cortical stimulation. Neurology. 1999;53(9):2073.

53. Motamedi G, rRP L, Miglioretti DL, Mizuno-Matsumoto Y, Gordon B, WRS W, et al. optimizing parameters for terminating cortical Afterdischarges with pulse stimulation. Epilepsia. 2002;43(8):836-46.

54. Pediaditis M, Tsiknakis $M$, Leitgeb N. Vision-based motion detection, analysis and recognition of epileptic seizures - a systematic review. Comput Methods Prog Biomed. 2012;108(3):1133-48.

55. Freestone DR, Kuhlmann L, Grayden DB, Burkitt AN, Lai A, Nelson TS, et al. Electrical probing of cortical excitability in patients with epilepsy. Epilepsy Behav. 2011:22:S110-S8.

56. Weinand ME, Carter LP, el-Saadany WF, Sioutos PJ, Labiner DM, Oommen KJ. Cerebral blood flow and temporal lobe epileptogenicity. J Neurosurg. 1997;86(2):226-32.

57. Patel KS, Zhao M, Ma H, Schwartz TH. Imaging preictal hemodynamic changes in neocortical epilepsy. Neurosurg Focus. 2013;34(4):E10.

58. Ákos SC, ML C, KK M, LL D, LO V, Michael G, et al. Electromyography-based seizure detector: preliminary results comparing a generalized tonic-clonic seizure detection algorithm to video-EEG recordings. Epilepsia. 2015;56(9): 1432-7.

59. Larsen SN, Conradsen I, Beniczky S, HBD S. Detection of tonic epileptic seizures based on surface electromyography. In: 2014 36th Annual International Conference of the IEEE Engineering in Medicine and Biology Society; 2014

60. Rajna P, Clemens B, Csibri E, Dobos E, Geregely A, Gottschal M, et al. Hungarian multicentre epidemiologic study of the warning and initial symptoms (prodrome, aura) of epileptic seizures. Seizure. 1997;6(5):361-8.

61. Lohse A, Kjaer TW, Sabers A, Wolf P. Epileptic aura and perception of selfcontrol. Epilepsy Behav. 2015;45:191-4.
62. Teixeira CA, Direito B, Feldwisch-Drentrup H, Valderrama M, Costa RP, Alvarado-Rojas C, et al. EPILAB: a software package for studies on the prediction of epileptic seizures. J Neurosci Methods. 2011;200(2):257-71.

63. Dourado A, Teixeira C, Quyen MLV, Schelter B, Favaro G, Schulz-Bonhage A, et al., editors. Giving hope to refractory epileptic patients. 2014 IST-Africa Conference Proceedings; 2014.

64. Greene BR, Boylan GB, Reilly RB, de Chazal P, Connolly S. Combination of EEG and ECG for improved automatic neonatal seizure detection. Clin Neurophysiol. 2007;118(6):1348-59.

65. Qaraqe M, Ismail M, Serpedin E, Zulfi H. Epileptic seizure onset detection based on EEG and ECG data fusion. Epilepsy Behav. 2016;58:48-60.

66. Van de Vel A, Verhaert K, Ceulemans B. Critical evaluation of four different seizure detection systems tested on one patient with focal and generalized tonic and clonic seizures. Epilepsy Behav. 2014;37:91-4.

67. Francesco O, Giulia R, Chiara C, Matteo M, Daniel B, Ming-Zher P, et al. Multicenter clinical assessment of improved wearable multimodal convulsive seizure detectors. Epilepsia. 2017;58(11):1870-9.

68. Conradsen I, Beniczky S, Wolf P, Kjaer TW, Sams T, Sorensen HBD. Automatic multi-modal intelligent seizure acquisition (MISA) system for detection of motor seizures from electromyographic data and motion data. Comput Methods Prog Biomed. 2012;107(2):97-110.

69. Schulze-Bonhage A. Long-term outcome in neurostimulation of epilepsy. Epilepsy Behav. 2019;91:25-29.

70. Schulze-Bonhage A. Brain stimulation as a neuromodulatory epilepsy therapy. Seizure. 2017;44:169-75.

71. Beddoe AE. Epilepsy: treatment using vagal nerve stimulation. In: Pravikoff D, editor. . Ipswich: EBSCO Publishing; 2018.

72. Cukiert A, Rydenhag B, Giordano F, Zicca A, Barba C, Guerrini R, et al. Vagus nerve stimulation: surgical technique of implantation and revision and related morbidity. Epilepsia. 2017; \$1:85.

73. Ching J, Khan S, Reed J, Ramnarine D, Sandeman D, Sieradzan K, et al. Long-term effectiveness and tolerability of vagal nerve stimulation in adults with intractable epilepsy: a retrospective analysis of 100 patients. $\mathrm{Br} \mathrm{J}$ Neurosurg. 2013;27(2):228-34.

74. Ben-Menachem E, Revesz D, Simon BJ, Silberstein S. Surgically implanted and non-invasive vagus nerve stimulation: a review of efficacy, safety and tolerability. Eur J Neurol. 2015;22(9):1260-8.

75. Cukiert A, Rydenhag B, Lehtimaki K. Deep brain stimulation targeting in refractory epilepsy. Epilepsia. 2017;\$1:80.

76. Schulze-Bonhage A. Deep brain stimulation: a new approach to the treatment of epilepsy. Dtsch Arztebl Int. 2009;106(24):407-12.

77. van der Vlis TAM B, OEMG S, FLWJ S, Hoogland G, Kubben P, Wagner L, et al. Deep brain stimulation of the anterior nucleus of the thalamus for drug-resistant epilepsy. Neurosurg Rev. 2019;42(2):287-96.

78. Hupalo M, Wojcik R, Jaskolski DJ. Intracerebral electroencephalography in targeting anterior thalamic nucleus for deep brain stimulation in refractory epilepsy. Neurol Neurochir Pol. 2018;52(3):379-85.

79. Halpern $\mathrm{CH}$, Samadani U, Litt B, Jaggi JL, Baltuch $\mathrm{GH}$. Deep brain stimulation for epilepsy. Neurotherapeutics. 2008;5(1):59-67.

80. SceneRay. Deep Brain Stimulation. In: Ltd SC, editor. 2014.

81. Salanova V, Witt T, Worth R, Henry TR, Gross RE, Nazzaro JM, et al. Longterm efficacy and safety of thalamic stimulation for drug-resistant partial epilepsy. Neurology. 2015;84(10):1017-25.

82. FDA USFaDA. Medtronic DBS System for Epilepsy - P960009/S219 2018 [updated 03/05/2018. Available from: https://www.fda.gov/MedicalDevices/ ProductsandMedicalProcedures/DeviceApprovalsandClearances/RecentlyApprovedDevices/ucm606550.htm. Accessed 25 Oct 2019.

83. Klinger NV, Mittal S. Clinical efficacy of deep brain stimulation for the treatment of medically refractory epilepsy. Clin Neurol Neurosurg. 2016;140:11-25.

84. Thomas GP, Jobst BC. Critical review of the responsive neurostimulator system for epilepsy, vol. 8. Auckland: Medical Devices; 2015. p. 405-11.

85. Morrell MJ. Responsive cortical stimulation for the treatment of medically intractable partial epilepsy. Neurology. 2011;77(13):1295-304. https://doi.org/ 10.1212/WNL.0b013e3182302056

86. Heck CN, King-Stephens D, Massey AD, Nair DR, Jobst BC, Barkley GL, et al. Two-year seizure reduction in adults with medically intractable partial onset epilepsy treated with responsive neurostimulation: final results of the RNS system pivotal trial. Epilepsia. 2014;55(3):432-41.

87. Bergey GK, Morrell MJ, Mizrahi EM, Goldman A, King-Stephens D, Nair D, et al. Long-term treatment with responsive brain stimulation in adults with refractory partial seizures. Neurology. 2015;84(8):810-7. 
88. GE B, ST L, GR E, GR R, BG L, BC W, et al. Brain-responsive neurostimulation in patients with medically intractable mesial temporal lobe epilepsy. Epilepsia. 2017;58(6):994-1004.

89. Pereira LS, Müller VT, da Mota GM, Rotenberg A, Fregni F. Safety of repetitive transcranial magnetic stimulation in patients with epilepsy: a systematic review. Epilepsy Behav. 2016:57:167-76.

90. Nitsche MA, Paulus W. Noninvasive brain stimulation protocols in the treatment of epilepsy: current state and perspectives. Neurotherapeutics. 2009;6(2):244-50

91. Carrette S, Boon P, Dekeyser C, Klooster DCW, Carrette E, Meurs A, et al. Repetitive transcranial magnetic stimulation for the treatment of refractory epilepsy. Expert Rev Neurother. 2016;16(9):1093-110.

92. San-Juan D, Espinoza López DA, Vázquez Gregorio R, Trenado C, FernándezGonzález Aragón M, Morales-Quezada L, et al. Transcranial direct current stimulation in mesial temporal lobe epilepsy and hippocampal sclerosis. Brain Stimul. 2017;10(1):28-35.

93. Assenza G, Campana C, Assenza F, Pellegrino G, Di Pino G, Fabrizio E, et al. Cathodal transcranial direct current stimulation reduces seizure frequency in adults with drug-resistant temporal lobe epilepsy: a sham controlled study. Brain Stimul. 2017;10(2):333-5.

94. Dietrich S, Smith J, Scherzinger C, Hofmann-Preiß K, Freitag T, Eisenkolb A, et al. A novel transcutaneous vagus nerve stimulation leads to brainstem and cerebral activations measured by functional MRI Funktionelle

Magnetresonanztomographie zeigt Aktivierungen des Hirnstamms und weiterer zerebraler Strukturen unter transkutaner Vagusnervstimulation 2008. 104-111 p.

95. He W, Jing X, Wang X, Rong P, Li L, Shi H, et al. Transcutaneous auricular vagus nerve stimulation as a complementary therapy for pediatric epilepsy: a pilot trial. Epilepsy Behav. 2013;28(3):343-6.

96. Bauer S, Baier H, Baumgartner C, Bohlmann K, Fauser S, Graf W, et al. Transcutaneous Vagus nerve stimulation (tVNS) for treatment of drugresistant epilepsy: a randomized, double-blind clinical trial (cMPsE02). Brain Stimul. 2016;9(3):356-63.

97. Fanselow EE. Central mechanisms of cranial nerve stimulation for epilepsy Surg Neurol Int. 2012;3(Suppl 4):S247-S54.

98. DeGiorgio CM, Soss J, Cook IA, Markovic D, Gornbein J, Murray D, et al. Randomized controlled trial of trigeminal nerve stimulation for drugresistant epilepsy. Neurology. 2013;80(9):786-91.

99. DC M, Alan S, Diane M, Todd W. Pilot study of trigeminal nerve stimulation (TNS) for epilepsy: a proof-of-concept trial. Epilepsia. 2006;47(7):1213-5.

100. Zare M, Salehi M, Mahvari J, Najafi MR, Moradi A, Pour MH, et al. Trigeminal nerve stimulation: a new way of treatment of refractory seizures. Adv Biomed Res. 2014;3:81

101. Berényi A, Belluscio M, Mao D, Buzsáki G. Closed-loop control of epilepsy by Transcranial electrical stimulation. Science. 2012;337(6095):735-7.

102. Sun FT, Morrell MJ. Closed-loop Neurostimulation: the clinical experience. Neurotherapeutics. 2014;11(3):553-63.

103. Premarket Approval [Internet]. 2017. Available from: https://www.accessdata. fda.gov/scripts/cdrh/cfdocs/cfpma/pma.cfm?id=P970003S207. Accessed 25 Oct 2019.

104. Boon P, Vonck K, van Rijckevorsel K, Tahry RE, Elger CE, Mullatti N, et al. A prospective, multicenter study of cardiac-based seizure detection to activate vagus nerve stimulation. Seizure. 2015;32:52-61.

105. Hampel KG, Vatter H, Elger CE, Surges R. Cardiac-based vagus nerve stimulation reduced seizure duration in a patient with refractory epilepsy. Seizure. 2015;26:81-5.

106. Hamilton P, Soryal I, Dhahri P, Wimalachandra W, Leat A, Hughes D, et al. Clinical outcomes of VNS therapy with AspireSR ${ }^{\oplus}$ (including cardiac-based seizure detection) at a large complex epilepsy and surgery Centre. Seizure. 2018:58:120-6.

107. Liu C, Wen X-W, Ge Y, Chen N, Hu W-H, Zhang T, et al. Responsive neurostimulation for the treatment of medically intractable epilepsy. Brain Res Bull. 2013;39.

108. Morrell MJ. Responsive cortical stimulation for the treatment of medically intractable partial epilepsy. Neurology. 2011;77(13):1295-304.

109. NeuroPace. RNS System User Manual 2019, Revision 4. [User Manual]. Available from: https://www.neuropace.com/manuals/RNS_System_User_ Manual.pdf. Accessed 25 Oct 2019.

110. Geller EB, Skarpaas TL, Gross RE, Goodman RR, Barkley GL, Bazil CW, et al. Brain-responsive neurostimulation in patients with medically intractable mesial temporal lobe epilepsy. Epilepsia. 2017;6:994.
111. Shoeb A, Pang T, Guttag J, Schachter S. Non-invasive computerized system for automatically initiating vagus nerve stimulation following patientspecific detection of seizures or epileptiform discharges. Int J Neural Syst. 2009:19(3):157-72.

112. Alessandro MD, Esteller R, Vachtsevanos G, Hinson A, Echauz J, Litt B. Epileptic seizure prediction using hybrid feature selection over multiple intracranial EEG electrode contacts: a report of four patients. IEEE Trans Biomed Eng. 2003;50(5):603-15.

113. Gotman J. Automatic recognition of epileptic seizures in the EEG. Electroencephalogr Clin Neurophysiol. 1982;54(5):530-40.

114. Litt B, Esteller R, Alessandro MD, Echauz J, Shor R, Bowen C, et al., editors. Evolution of accumulated energy predicts seizures in mesial temporal lobe epilepsy. Proceedings of the First Joint BMES/EMBS Conference 1999 IEEE Engineering in Medicine and Biology 21st Annual Conference and the 1999 Annual Fall Meeting of the Biomedical Engineering Society (Cat N; 1999) 1999.

\section{Publisher's Note}

Springer Nature remains neutral with regard to jurisdictional claims in published maps and institutional affiliations.

Ready to submit your research? Choose BMC and benefit from:

- fast, convenient online submission

- thorough peer review by experienced researchers in your field

- rapid publication on acceptance

- support for research data, including large and complex data types

- gold Open Access which fosters wider collaboration and increased citations

- maximum visibility for your research: over $100 \mathrm{M}$ website views per year

At BMC, research is always in progress.

Learn more biomedcentral.com/submissions 